\title{
Safety and Automation of Collaborative Robot System in Work Environment
}

\author{
Richard Olawoyin* \\ Department of Environmental Health and Safety, Oakland University, USA
}

Submission: May 25, 2018; Published: July 10, 2018

*Corresponding author: Richard Olawoyin, Environmental Health and Safety, Oakland University-Rochester Michigan, 48309, USA, Email: olawoyin@aoakland.edu

Keywords: Safety and Automation; Collaborative robot system; Work Environment; Factors; Technological design; Industrial processes

Abbrevations: PA: Programmable Automation; ACF: Advanced Control Features; EDI: Enterprise Data Integration; RCI: Robotic Cells Interoperability; AI: Artificial Intelligence; PLC: Programmable Logic Controllers; SCS: Safety Critical System; ISO: International Organization for Standardization

\section{Summary}

A few factors are important for determining the effects of automation on the work environment, including the technological design and applications, approaches used for their presentation, and the automation goals of the organization's management team. The application of robotics and automation to industrial processes provide optimization benefits relative to the work processes, however, the potential also exist for new situations to develop that may introduce system risks and human psychological risks to the employees. The main goal is to plan, design and implement robotic and automation programs that will be beneficial to the workforce, improving productivity, safety and quality.

The application and implementation of modern industrial automation and robotics systems may potentially pose a challenge and perhaps unpromising combination of requirements. It is logical to expect that control systems must generally interface with sensor or actuator signals, which is germane to other modern applications. For most modern industrial applications, such as programmable automation (PA), advanced control features (ACF), enterprise data integration (EDI), robotic cells interoperability (RCI) and network connectivity, there has been increasing demand for their use. The innovative edge in industrial robotics and automation involve accessible and user-friendly collaborative robot (cobots) solutions[1], that allow the human workforce and robots cells to share skills towards achieving same goal The human workforce performance adds to this collaboration with the inherent cognitive skills and opportunities for flexibility, while the robotic systems bring high powered speed, accuracy, precision and repeatability. To efficiently optimize this collaborative set-up, there are a few challenges that should be addressed. All direct contacts between the workers and the operational robotic cells must be prevented to assure safe interaction and collaboration. Capitalizing on the benefits of human cognitive skills, it is crucial to correctly plan and design all spontaneous operator interfaces, with special considerations for safety critical systems, making it easy and safe for the operators to program and interact with the robotic cells.

\section{Introduction}

The global annual industrial robots supply is predicted to increase at the rate of $13 \%$ and it was estimated that 2.6 million industrial robots will be operational by 2019 [2], with highest demands in the automotive and electronic industries. Other small and medium scale companies are beginning to also employ the collaborative solutions of using both robots and human skills. Majority of the design and development efforts for human-friendly, safe and adaptable automation and robotic are from industrial manufacturing. To be at the competitive advantage in today's marketplace, the manufacturing industry rely on automation and robotics to stay ahead. The concept of robots can be traced as far back as to the beginning of civilization. Aristotle conceptualized in 322 B.C., the emergence of an intelligent tool that "when ordered or even of its own accord, could do the work that befits it." Fast forward to the 20 century, the 1960's marked the emergence of industrial robots on assembly lines of America's automobile manufacturing companies. Currently, there are several major advancements and technological breakthroughs that have integrated control systems and automation innovations, artificial intelligence 


\section{Robotics \& Automation Engineering Journal}

(AI) methodologies and microprocessors, to optimize the functionality of robots, expanding to other fields such as medicine, agriculture, transportation etc. These technological advancements in the design of automated robots have not been able to completely eradicate the potential for risks for cobot and traditional robot (trabot), see Figure 1. A fault to a safety critical system of a robot can pose fatal or serious injuries to humans and may also result to the loss of capital investments in the machines [3].

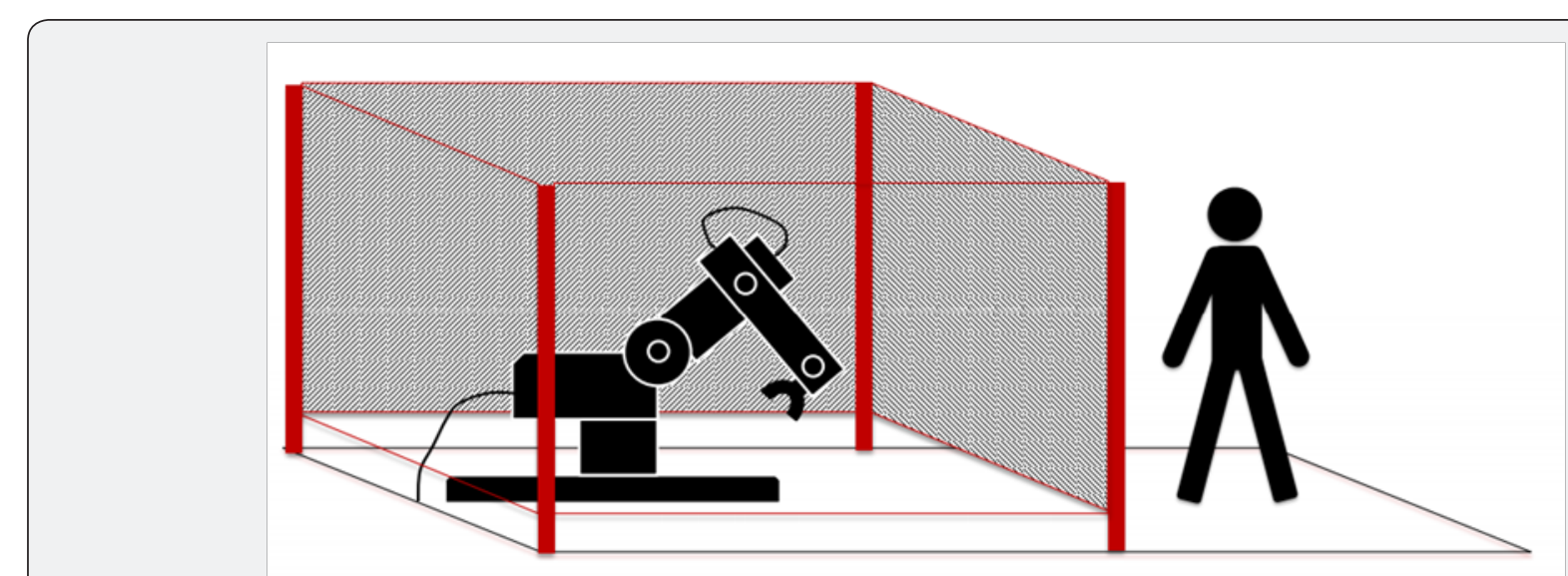

Figure 1: Traditional robot with safeguarding devices, prevents operators access to machine. (Courtesy Robotics Industries Association).

\section{Safety Critical Systems}

A Safety Critical System (SCS) is a system that can potentially cause accidents directly or indirectly, once failed. These failures can potentially causefatalities, environmental damages and property/financial losses. The overall system must be safe which depends on proper operations of the robot and examples of SCS include; production industries (manufacturing controls, interlocking systems, maintenance and application of robotic cells), process industry (chemical process control, energy and power generation etc.), medicine (medical robotic cell controls, radiation therapy systems etc.). Identification systems, programmable logic controllers (PLC), industrial and power controls systems, sensor systems are examples of automation technologies currently widely used. The automation of SCS operationsinvolved embedded control systems which applies to both the hardware and software components including the devices, equipment, electrical and mechanical components. Whenever these software and hardware are employed for SCS control, there is a potential for these components to pose imminent hazards to operators or anyone in the zone of exposure of the systems. The automation is dependent on the software capabilities and the proper development and design of the software will produce efficient operation with capabilities of anticipating, detecting and eliminating potential hazards that may contribute to software errors of the SCS, hence protecting people, property and the environment.

The complexities, sophistication and requirements for robotic systems are addressed for the automation and robotics industry through the International Organization for
Standardization (ISO) for robots and robot systems integration. ISO 10218-1:2006 - specifies the requirements and guidance for the assurance of safety in design and construction of the robot only (manipulator and controller) and not the overall robot system. The standard was updated in 2011 as Part 2 or ISO 10218-2:2011, which includes the robot system/cell and application. It also covers the integration and installation of robot system or cell, which therefore means that it provides a more comprehensive set of requirements for robot safety. ISO TS 15066:2016 provides the technical specification of safety requirements for collaborative industrial robot systems in the work environment and it also provides supplementary requirements and guidance on collaborative industrial robot operations (does not apply to non-industrial robots) in the ISO 10218-1:2006 and ISO 10218-2:2011. ANSI/RIA R15.06-2012 is an adaptation of the 10218-2:2011.

\section{Industry 4.0Initiative and Intuitive User Interfaces}

In today's high-speed high accuracy economy, manufacturing companies continue to drive for increased efficiency, production speed, quality and workforce safety, cobot can be significantly effective in protecting workers, at same time, increasing outputs and decreasing the operational costs and associated expenses. In manufacturing technologies, the contemporary trend in automation and data exchange which makes production system smarter is known as Industry 4.0 and it also involves the use of collaborative robotic solutions [4]. Industry 4.0 presents transparency which is crucial for efficient supply chain. The benefits of industry 4.0 are as follows; 
a) Process efficiency: Automation, robotics and digitalization reduce redundancy and increase flexibility through optimization solutions.

b) Increased production and quality: The product quantity and quality can be enhanced throughintegrated automation, real-time monitoring and quick intervention for the correction of system errors.

c) Cost reduction and energy savings: Industry 4.0 increases overall process efficiency which in turn, yields direct energy and cost savings.

d) Less human intervention: With robots taking over repetitive manual jobs, humans can be used for more skillful tasks.

e) Competitive advantage: Industry $4.0 \mathrm{is}$ promising to become indispensable and companies that adopt this strategy stand to gain and become a leader in the industry.

f) Personalization: Products can be safely and quickly made based on thecustomer'sneeds and current trends.

The application of cobot for industrial processes is valuablesince the system can be trained through intuitive systems, usingprogramming by demonstration [5], augmented reality [6], walkthrough programming [7] etc. In contrast, traditional non-cobots usually require experts to program the robotic cell using the conventional programming methodologies. There are challenges involved with the use of robots for industrial processes, one of which is often associated with operator interactions with the robot, since it typically entails specialized knowledge. Equally, the availability of intuitive procedures of interactions with robots through proper programming is a significant enabler for continuousimplementation of automation and robotic technologies, which would also benefit small and medium sized companies. Easymethods of interactions with industrial robots in a timely fashion that also reducesoperator's errors and preserves situational awareness, are required.

\section{Optimizing Safety with Smarter Robots}

Safety is crucial for products and system designs particularly at collaborative work areas, where robots operate alongside humans. The safety and reliability of robotic systems are integral to optimal solution developments for future practical challenges in robotic design for human situations [8]. The developmentand evolution of automation processes and robotics are crucial to the changing world. The first developed industrial robots were huge, hydraulically powered machines. The early robots lacked intelligence without the operator's assistance, though they functioned withsubstantial strength. Industrial robots evolved into electrically powered standalone units, with enhancedperformance and accuracy in the 1980's.
The continuous improvements to microprocessors and innovations to automated control technologies have positioned robots as the center-piece tool for industrial revolution.

\section{Human-Robot Collaboration (HrC)}

The human-robots interaction is being transformed due to the advent of innovative software and SCS functionality [9]. Conventionally, people were protected from being hurt, injured or killed from the action of a moving robot, by using either of the hazard control methods that include elimination, substitution, warnings, engineering control (safe guards and barriers), administrative control (e.g. training) or protective equipment. Whenever an operator interfaces with the robot (for instance loading or unloading of materials), safety control systems (such as., light screen, safety mat, other presence sensing devices) would have to be in place guarantee that the robot is in a safety position and state. Basically, the robot's motion is conditioned and eventually, the energy source is removed, and the robot then stops completely. The effect of which slows down production. Innovations in software-based SCS have produced safe-speed core technology that allows for the manipulation of the robot's operational motion, so that humans can share the workspace together. In the same work area, robots could bring heavy slabs closer, while the skilled operator marks them, though this process would have to take place only if there are environmental sensors or presence sensing sensors in the work zone. Never technologies now also allow for Robot-to-Robot Synchronization(r2rS). This happens when one robot is programmed to coordinate the actions of multiple other robots. The combination of $\mathrm{HrC}$ and $\mathrm{r} 2 \mathrm{rS}$ can significantly reduce down time, increase productivity and further enhances safety.

\section{Conclusion and future directions of research}

To address safety related issues for automation and robotics, it is important to consider optimizing performance relative to the limits of safety rather than classifying safety as a criterion that limits performance, therefore the use of performance-oriented solutions should be desired.

Furthermore, enhancing the effectiveness of $\mathrm{HrC}$ in actual industrial situations is the primerfor adaptive solutions for comprehensive robotics [10]. Animpendinggoal in the research and development community is to automate robots and equip them with suitable cognitive processing skills and collectiveindependent proficiencies, thereforereleasing or complimenting thecognitive efforts of human operators, particularly in intricate tasks and situations.

\section{References}

1. Colgate J, Wannasuphoprasit W, Peshkin MA (1996) Cobots: robots for collaboration with human operators. Proceedings of the international mechanical engineering congress and exhibition 58: 433-439. 
2. IFR (2016) Executive summary world robotics 2016 industrial robots Technical Report. International Federation of Robotics (IFR).

3. Harper C, Virk G (2010) Towards the development of international safety standards for human robot interaction. Int J Social Robotics 2(3): 229-234

4. Kagermann H, Helbig J, Hellinger A, Wahlster W (2013) Recommendations for implementing the strategic initiative industrie 4.0: securing the future of german manufacturing industry; final report of the industrie 4.0 working group. Technical Report. Acatech - National Academy of Science and Engineering.

5. Billard AG, Calinon S, Dillmann R (2016) Learning from humans. In: Siciliano B, Khatib 0 (Eds.), Springer handbook of robotics. 2nd ed. Springer, pp. 1995-2014.

6. Lambrecht J, Kleinsorge M, Rosenstrauch M, Krüger J (2013) Spatial programming for industrial robots through task demonstration. Int Adv Rob Syst 10(5): 254
7. Farsoni S, Landi CT, Ferraguti F, Secchi C, Bonfè M (2017) Compensation of load dynamics for admittance controlled interactive industrial robots using a quaternion based Kalman filter. IEEE Robot Autom Lett 2(2): 672-679.

8. De Santis A, Siciliano B, De Luca A, Bicchi A (2008) An atlas of physical human-robot interaction. Mech Mach Theory 43(3): 253-270.

9. Ding H, Schipper M, Matthias B (2014) Optimized task distribution for industrial assembly in mixed human-robot environments-case study on IO module assembly. Proceedings of the IEEE international conference on automation science and engineering (CASE), IEEE 19-24.

10. Villani V, Sabattini L, Czerniak JN, Mertens A, Fantuzzi C (2018) MATE Robots simplifying my work: benefits and socio-ethical implications. IEEE Robot Autom Mag. 